\title{
A control approach for transitioning VTOL UAVs with continuously varying transition angle and controlled by differential thrust
}

\author{
B. Theys*, G. De Vos, J. De Schutter \\ KU Leuven, Leuven, Belgium
}

\begin{abstract}
This paper describes a control approach for transitioning VTOL UAVs controlled by differential thrust, allowing to fly in all flight phases spanning a continuous transition between hover and cruise flight. A quaternion based attitude controller makes use of an additional reference frame that is rotated by the transition angle with respect to the body-fixed frame. This approach allows intuitive control and applies to various vehicle configurations. After building a simulation model, a small series of tests is used to build and validate maps of the flight characteristics of the vehicle. These maps are inverted and used to control the vehicle's velocity. Test flights with a custom built VTOL UAV for transition angles up to $70^{\circ}$, validate the control approach.
\end{abstract}

\section{INTRODUCTION}

Currently, UAVs are increasingly deployed in various applications such as surveillance, mapping, inspection or transportation. VTOL UAVs such as multi-rotors prove to be advantageous over conventional fixed-wing UAVs due to their ability to land and take-off vertically or hover. The disadvantage is their limited flight speed and distance. Therefore, VTOL UAVs that can transition from hover to efficient forward flight are being developed such as reported by [1]. Control is a major challenge for these transitioning vehicles since they have very distinct flight phases. On top of that, various configurations of propellers, control surfaces or other actuators are used in the design of transitioning VTOL UAVs. Hence, different controllers might be required for each design configuration. Figure 1 presents an overview of different configurations of VTOL UAVs controlled by differential thrust. Configuration (a) uses two wings, in the prop wash of the quadcopter [2]. A ' + ' - configuration is used for configuration (b) [3] and configuration (c) [4]. This provides a structural advantage, since the arms connecting the propellers also strengthen the wing. The swirl of the wake of the two propellers at the wing tips can also potentially counteract the

*Email address: bart.theys@kuleuven.be swirl behind the wing tips, thereby improving aerodynamic efficiency. An annular wing is also possible, as presented in configuration (d) [5] and studied by [6]. The annular wing protects the propellers and decreases the noise. The VertiKUL (e), uses a ' $\mathrm{H}$ ' - configuration as internal structure to provide a large space for payload in the center of the vehicle. Its successor, in configuration (f), uses smaller wings to improve stability in windy conditions. The propellers therefore still produce part of the required lift in forward flight and are placed at an angle of $45^{\circ}$ with respect to the wing, in contrast to the former examples in which wing and propeller are placed at approximately $90^{\circ}$ with respect to each other. (a)

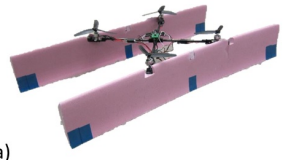

(c)

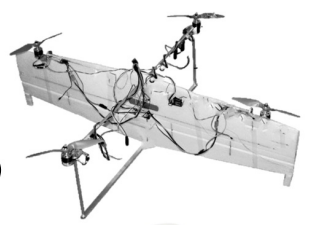

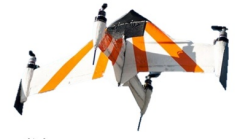

(b)

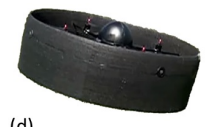

(d)

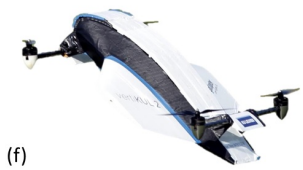

(f) (e)

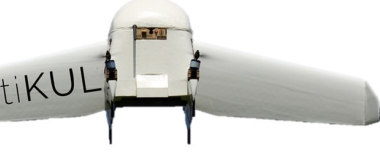

Fig. 1: Overview of transitioning VTOL UAVs configurations, controlled by differential thrust: (a) QuadRotor-Biplane, (b) XCraft XPlusOne, (c) QuadrotorTailsitter, (d) Santos Lab Orbis, (e) KU Leuven VertiKUL, (f) KU Leuven VertiKUL 2

A broad overview of other hybrid UAV designs, their modelling and used control strategies, is presented by Saeed et. al. [7]. This overview also includes designs with control surfaces. To control a transitioning UAV, initially a separate controller can be designed for both the hover phase and the cruise phase, with an automated transitioning manoeuvre between these two flight phases, as described by [8]. How- 
ever, this preludes the vehicle's potential of flying at intermediate speeds as in [9] and [10], where horizontal speed during cruise flight is fixed. Continuous flight in-between hover and cruise has been reported also by Johnson [11] and Wagter [12]. Wagter describes control of speed and altitude of the Atmos VTOL UAV and uses a clever interpolation between commands of different controllers. The control approach for transitioning VTOL UAVs, described in this paper follows from requirements at separate levels of control.

The first level of control is attitude control. Conventional roll-pitch-yaw with collective thrust as used for multi-rotors is very well suited when roll and pitch angles are limited, typically to + and $-45^{\circ}$. Most transitioning VTOL UAVs however are designed to pitch down up to $90^{\circ}$, which poses an interpretation problem for a human operator, as illustrated in figure 2: the front side of the vehicle points downwards after transition. Since the vehicle does not fly towards the ground in this new configuration, what used to be the upside of the vehicle is now being interpreted as the new front side. Additionally, pitching down towards $90^{\circ}$, results in a closeto-singularity representation of the roll-pitch-yaw representation of the orientation.
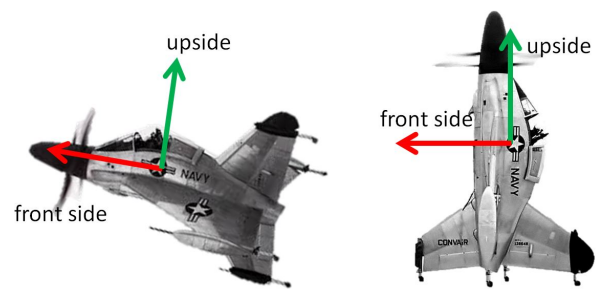

Fig. 2: Illustration of the interpretation problem after transition of the vehicle. (image: Convair XFY-1 Pogo)

The second level of control involves velocity control. The velocity control level allows inexperienced users to fly the vehicle with only three inputs: horizontal speed $V_{x}$, vertical speed $V_{z}$ and turning rate $\omega_{z}$, schematically presented in figure 3.

For fixed-wing UAVs, the horizontal speed is controlled with the throttle and the vertical speed with the pitch angle. For multi-copters, this is exactly the opposite: since the propellers point upward, vertical speed is controlled by throttle and horizontal speed by pitch angle as presented in figure 4 .

For transitioning VTOL UAVs, controlling horizontal speed or vertical speed involves a combination of pitch and throttle control action, depending on the transition angle they are flying at. In some controllers for multi-copters, thrust is scaled by a factor $1 / \cos \theta$, with $\theta$ the pitch angle, to compensate for the loss in lift at high horizontal speeds, hence high pitch angles. The same approach can be applied to fixedwing controllers: thrust is increased by some factor to compensate for the loss of forward velocity when a greater vertical speed is requested by increasing the pitch angle. This

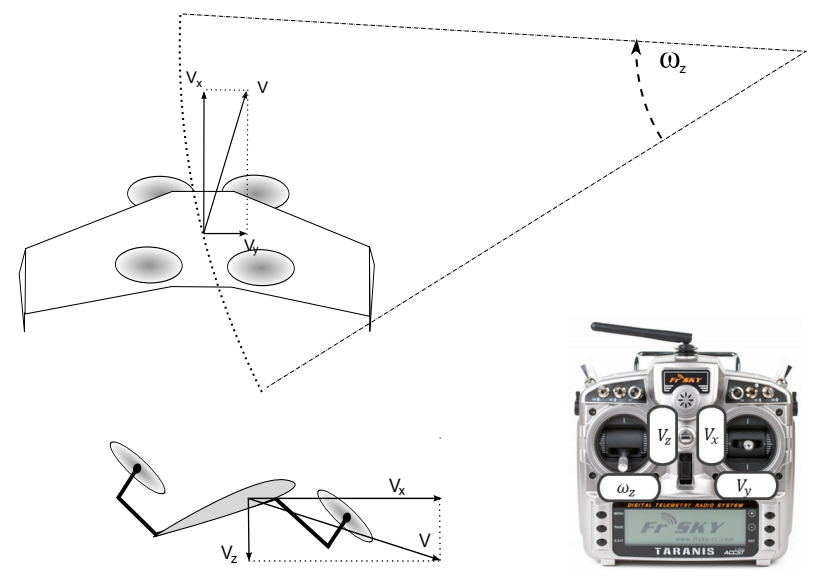

Fig. 3: Velocity control inputs and conventions.

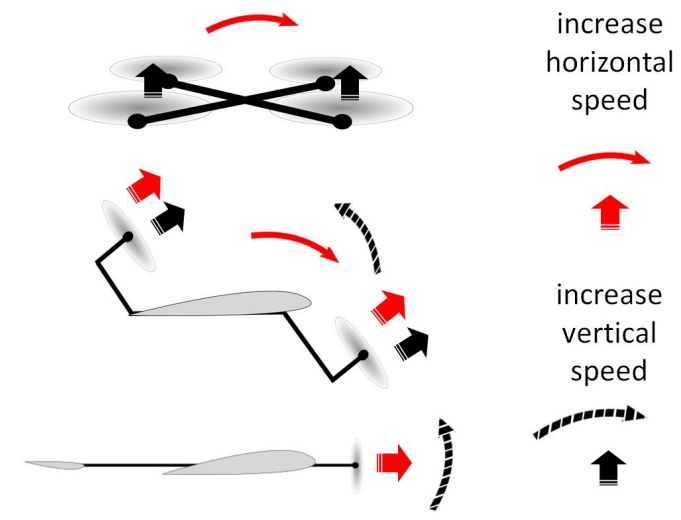

Fig. 4: For a VTOL UAV in hover or a fixed wing in cruise flight, horizontal and vertical speed control are performed by pitch or thrust control action. For transitioning VTOL UAVs, where the propellers have an angle with respect to the free-stream airflow, horizontal and vertical speed control are performed by a combination of pitch and thrust control action.

approach works fine for small changes in flight regime.

The third level of control finally uses $V_{x}, V_{z}$ and $\omega_{z}$ to generate a trajectory for the UAV. Such a navigation algorithm for a transitioning UAV is described by Ferrel [13]. In this paper, only the first two levels of control, i.e. attitude and velocity control, are described.

To test the control approach, a test vehicle is composed out of a quadcopter frame and a styrofoam wing, as presented in figure 5. The different components are specified in table 1 . The take-off mass of the test vehicle is $1.7 \mathrm{~kg}$ and the wingspan is $1.4 \mathrm{~m}$. The wings have a sweep of $23^{\circ}$ and winglets are installed for lateral stability. The motors are turned $10^{\circ}$ around the quadrotor arms to improve control capability for yaw in hover and roll in forward flight. The wing is mounted at an angle of $70^{\circ}$ with the propeller plane, thus resulting in a $0^{\circ}$ angle of attack at $70^{\circ}$ transition. 


\begin{tabular}{l|l}
\hline Component & Specification \\
\hline Frame & DJI F450 Flamewheel \\
\hline Battery & Zippy Flightmax 2800mAh \\
& 14,8V 4S 30C \\
& High discharge LiPo battery \\
\hline Propellers & DJI 8045R \\
\hline Motors & DJI 2212/920KV \\
\hline ESC & 30A OPTO \\
& 11,1V - 14,7V \\
& 3S-4S LiPo \\
& 30-450 Hz frequency respons \\
\hline Flight control & 3DR Pixhawk \\
\hline RC receiver & Robbe R6107SP \\
& 7 channel receiver \\
& 2,4GHz RASST \\
\hline GPS & 3DR uBlox GPS \\
\hline
\end{tabular}

Tab. 1: Overview of the components for the test vehicle

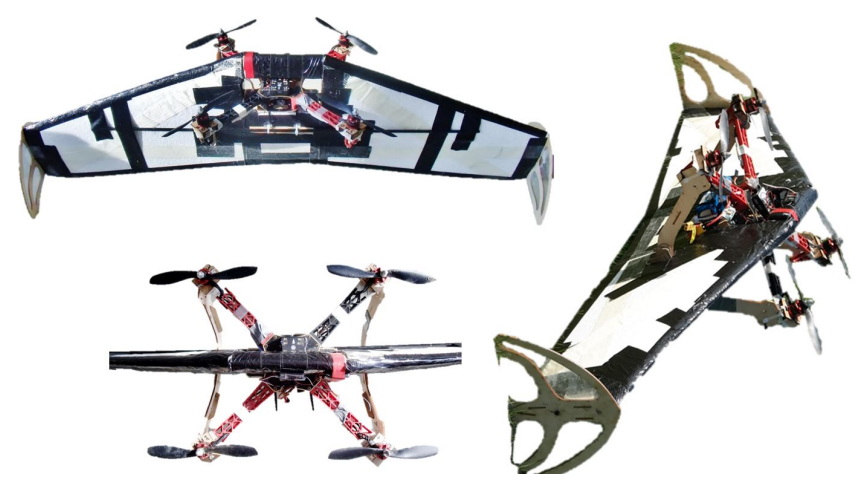

Fig. 5: The test vehicle.

\section{AtTitude CONTROL}

When flown by a human operator, the most common way to control a UAV is by controlling the roll and pitch angle, yaw rate and thrust. A feedback controller regulates the motor speeds in order to realize the desired attitude of the vehicle. The attitude of the vehicle with respect to the Earth is expressed as the orientation of a body-fixed coordinate frame attached to the UAV, with respect to the inertial frame, attached to the Earth and presented with rotation matrix ${ }_{w}^{b} R$. Because for human interpretation, the front side of the vehicle changes orientation after transition, an additional frame is defined for which the human operator can control roll, pitch and yaw with respect to the Earth frame. This frame ${ }_{w}^{t} R$ is rotated with respect to the body-fixed frame with an angle $\lambda$, now referred to as transition angle. The orientation of the body-fixed frame with respect to the earth frame is therefore composed as:

$$
{ }_{w}^{b} R={ }_{w}^{t} R{ }_{t}^{b} R,
$$

with

$$
{ }_{w}^{t} R=R(Z, \psi) R(Y, \theta) R(X, \phi),
$$

and

$$
{ }_{t}^{b} R=R(-Y, \lambda) .
$$

The definitions of the reference frames is presented in figure 6 .

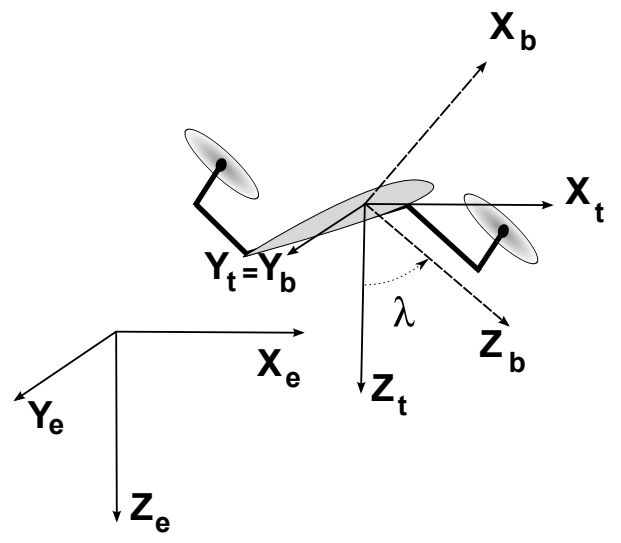

Fig. 6: Definition of the Earth frame, body-fixed frame, transition frame and transition angle.

The transition angle is also commanded by the human operator. This simple solution allows the operator to fly the vehicle intuitively at transition angles ranging from $0^{\circ}$ to $90^{\circ}$. Figure 7 schematically shows the addition of a transition angle to the attitude controller.

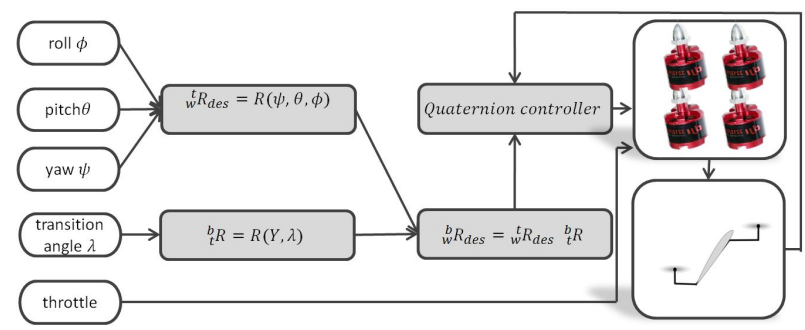

Fig. 7: Overview of the intuitive attitude control with use of an additional reference frame and transition angle.

\section{VELOCITY CONTROL}

The velocity controller is expected to allow speed and heading control by an inexperienced user or by a path planning algorithm. The controlled variables are the vehicle's horizontal speed $V_{x}$, vertical speed $V_{z}$, turning rate $\omega_{z}$ and, optional, lateral speed $V_{y}$, which is mainly interesting in the hover phase. The approach for velocity control proposed in this paper is based on a model of the response of the vehicle to a throttle and transition angle input. This model is then 
inverted and used to map $V_{x}$ and $V_{z}$ requirements into transition angle and throttle setting outputs. A similar approach, using a dynamic inversion method is presented by [14] and applied to a quad tilt wing UAV or by [15] for a transitioning fixed wing UAV.

In a first step a model of the test vehicle allows simulation of various flight phases between hover and forward flight, based on different control inputs. Instead of simulating the flight phases, they can also be measured during test flights as was done by Smeur [16] for the Quadshot VTOL UAV. A comparison with test flight data validates the simulation model. The velocity controller for $V_{x}$ and $V_{z}$ uses this model to regulate the thrust and transition angle. $\omega_{z}$ and $V_{y}$ are controlled with a control that does not require the model of the vehicle.

\subsection{Modelling}

The test vehicle is modelled in a Simulink environment. The model contains a finite wing with wing tips to model lift and directional stability. Airfoil data from $-180^{\circ}$ to $180^{\circ}$ angle of attack of the NACA 0012 airfoil is presented in figure 8 and used to model the wing. The parasite drag of the arms

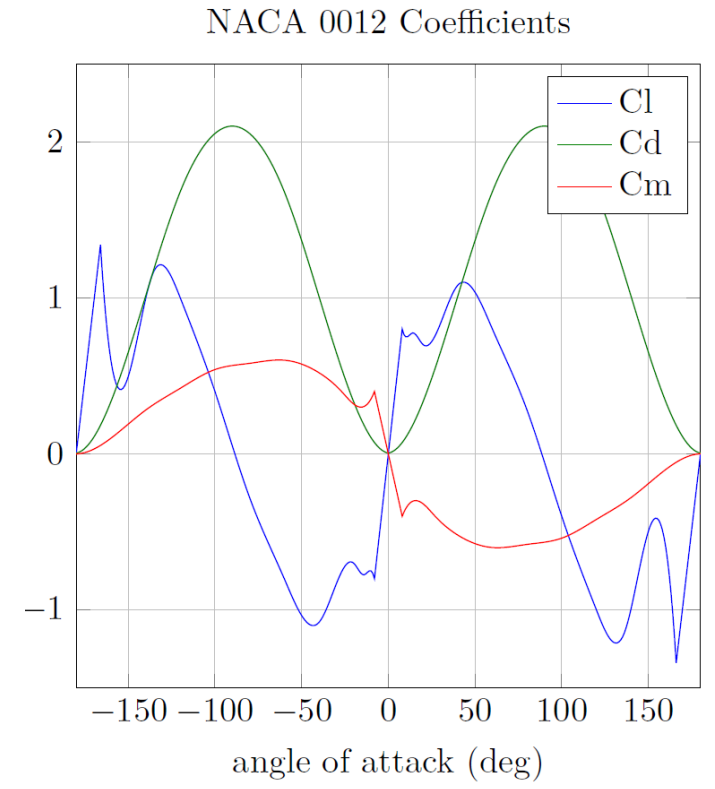

Fig. 8: Aerodynamic coefficients of a NACA 0012 airfoil [17].

and motors is approximated by the drag of a sphere around the center of gravity of the vehicle. The mass is measured and the inertia follows from a detailed 3D mass model. The thrust generated by the four propellers is modelled as a function of the throttle signal and the component of the flight speed parallel to the propeller shaft, or axial velocity $V_{a x}$. The propulsion system, consisting of battery, speed controller and motor for each of the four propellers, is tested with three different propellers to obtain the relation between $R P M, P W M$

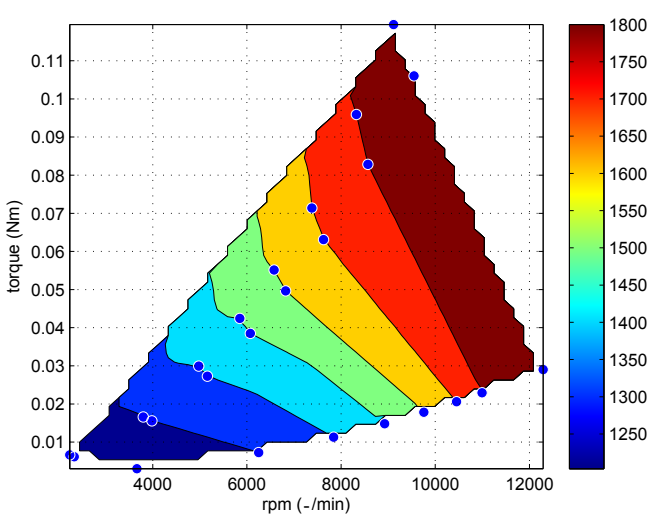

Fig. 9: Relation between torque, $R P M$ and $P W M$ throttle signal for the propulsion system of the test vehicle.

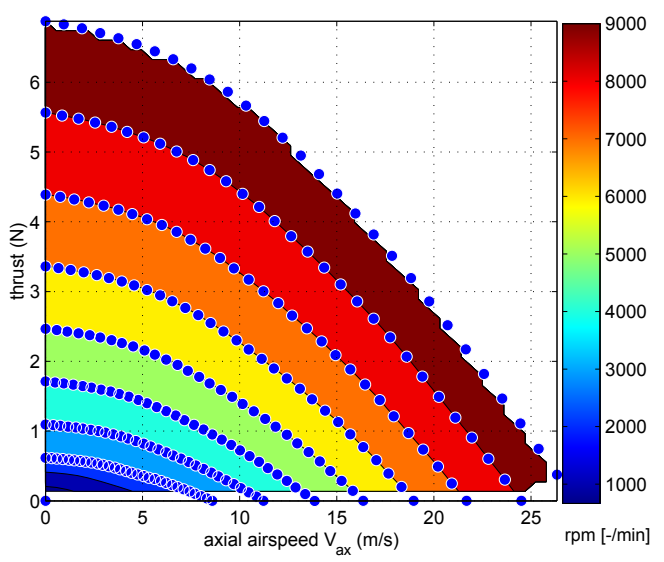

Fig. 10: $R P M$ of the propeller as a function of the axial air speed and required thrusts [18].

throttle signal and torque. Figure 9 presents this relation for the propulsion system of the test vehicle. The blue dots present $R P M$ and torque measurements for the three types of propellers. The relation between torque, $R P M$ and throttle is combined with propeller data presented in figure 10 and 11 to obtain the thrust as function of throttle and flight speed.

The steady state velocity $V_{x}$ and $V_{z}$ of the simulated vehicle with a constant throttle and transition angle applied, is recorded for the throttle varying between $0 \%$ and $100 \%$ and the transition angle between $-20^{\circ}$ and $90^{\circ}$. This results in two maps presented in figure 12 with, respectively, $V_{x}$ and $V_{z}$ as a function of the throttle and the transition angle. As expected and observed on the left graph, the highest speed $V_{x}$ is achieved at full throttle and the largest transition angle. At this operating point the vehicle is descending. The highest climbing rate is achieved also at full throttle at zero transition angle. At this operating point, the vehicle also has a small forward velocity. This is because the wing is now at a negative angle of attack, producing a force that pushes the vehicle forward. 


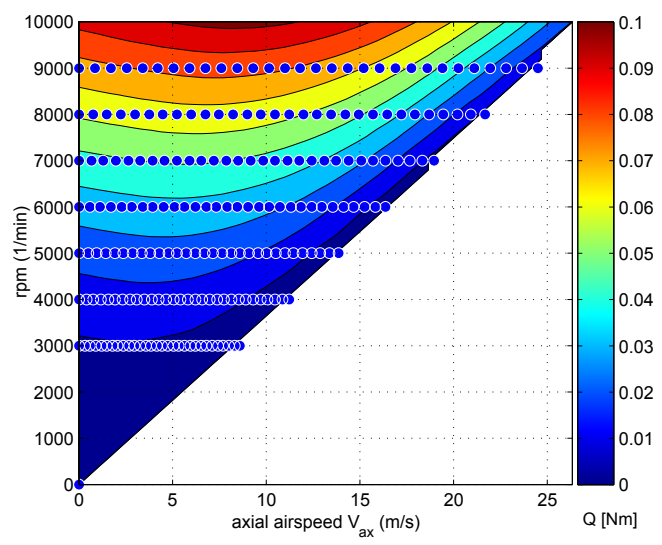

Fig. 11: Torque of the propeller as a function of the axial air speed and $R P M$ [18].
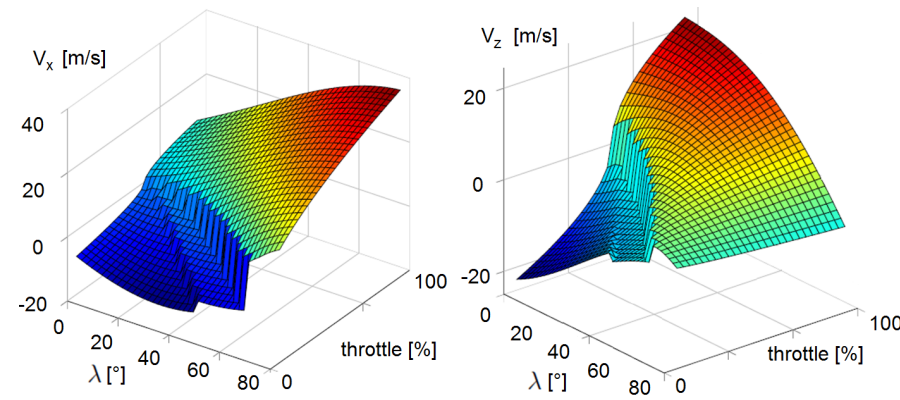

Fig. 12: Left: steady state $V_{x}$ of the test vehicle in simulation at a given throttle and transition angle. Right: steady state $V_{z}$ at a given throttle and transition angle.

Throttle and transition angle are required to accomplish the desired $V_{x}$ and $V_{z}$. Therefore the graphs are inverted and a smooth fit with a polynomial function is used to interpolate between the data points. The advantage of fitting a polynomial through the data points are that abrupt changes are smoothed and little memory and computing time is required to evaluate the function, in contrast to storing a large lookup table and interpolating between the values. The disadvantage is the loss of accuracy, depending on the quality of the fit.

To evaluate the polynomial fits that result from a series of simulations, they are compared to actual flight data, presented by the green dots on figure 13 .
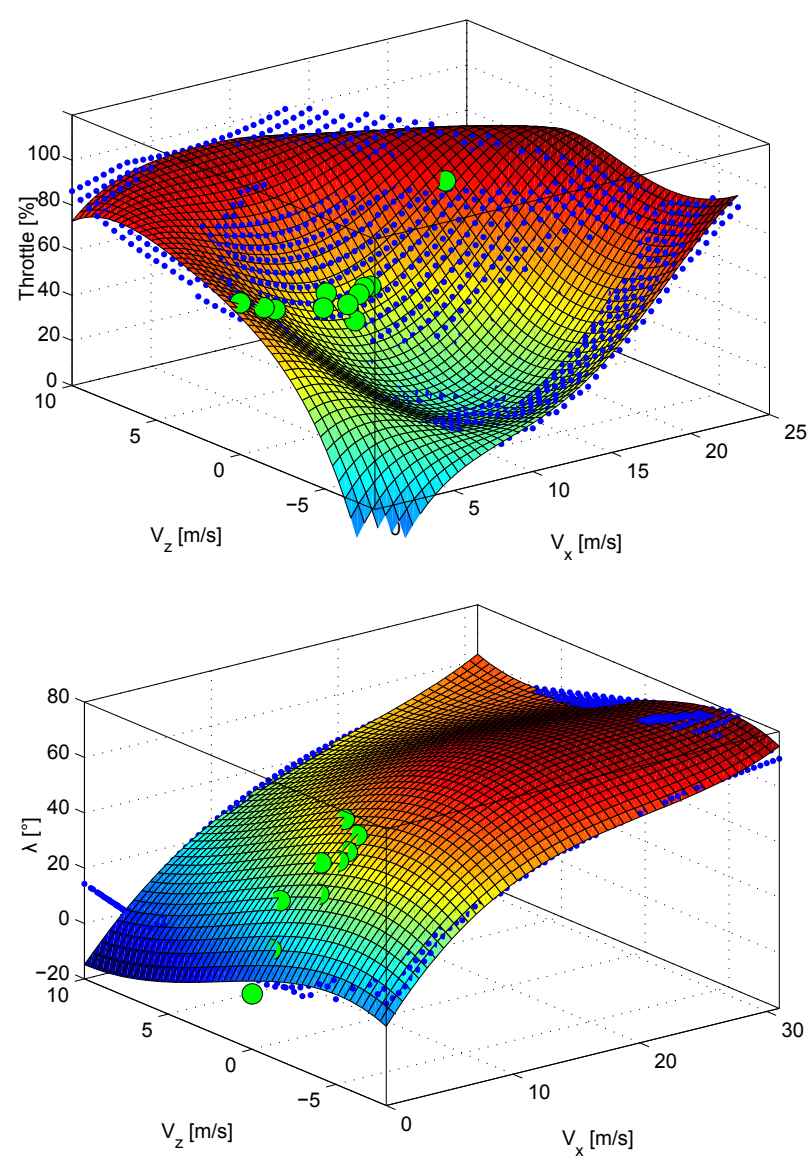

Fig. 13: Comparison between simulated data points, the fitted surface and measured data points in flight. The large green dots present data points from flight tests, the small blue dots present the simulated data points and a polynomial fit is presented by the surface. Top: amount of throttle required for steady state flight at a given $V_{x}$ and $V_{z}$. Bottom: required transition angle at a given $V_{x}$ and $V_{z}$.

\section{$3.2 V_{x}-V_{z}$ Control design}

With knowledge of the relation between the transition angle and throttle signal on one hand and the resulting horizontal and vertical velocity on the other hand, a controller is designed. For a desired flight speed, the appropriate throttle and transition angle are calculated based on the polynomials and provided as feed forward to the attitude controller. A PID controller is added to cancel the error between the desired and the measured velocities and decrease the response time between commanded velocity and actual velocity. Gain scheduling can be applied to improve the performance of the control loop [19]. When flying at high speed, typically a lower gain will be required for the altitude control, since a small control action in transition angle will result in a large change of vertical velocity. Without the additional controller, a step input from hover to fully transitioned forward flight 
would result in an initial loss of altitude since flight speed has to build up before the wing produces enough lift. Also, there will always remain an error between the polynomials used for the control and the reality. This is due to the imperfect simulation model, small mass and geometry changes on the vehicle after repairs or change of payload, external conditions such as wind and air density and the approximation of simulated operating points with a polynomial. Figure 14 shows the overview of the velocity controller, based on the polynomials shown in the center.

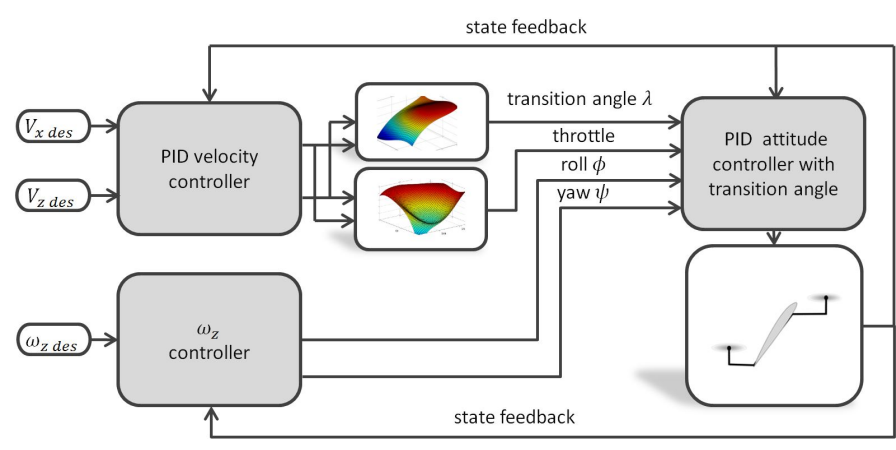

Fig. 14: Overview of the velocity controller

\section{$3.3 \omega_{z}$ Control design}

The heading of the vehicle is controlled by the $\omega_{z}$ controller. This controller accepts commands from the human operator or the path planner. The desired $\omega_{z}$ is integrated to find the reference heading angle $\psi_{\text {ref }}$. A roll angle is applied to cancel the lateral velocity of the vehicle and make a coordinated turn. The desired roll angle is calculated as [20]:

$$
\phi=\arctan \left(\frac{V \omega_{z}}{g}\right)
$$

with $V$ the velocity of the vehicle. This angle is applied as input to the roll controller, schematically presented in figure 15. A feedback loop cancels out the error on the lateral velocity. This controller minimizes the slip or skid angle, resulting in efficient turning behaviour. The maximum desired turning rate $\omega_{z \max }$ decreases for higher speeds since the ability of the vehicle to rapidly change heading decreases.

$$
\omega_{z \max }=\frac{g \tan \left(\phi_{\max }\right)}{V}
$$

This also keeps the maximum commanded roll angle $\phi_{\max }$ limited.

The user can still influence the roll angle with an additional term $\phi_{c t r l}$, presented in red on figure 15 , that allows the vehicle to fly with a lateral velocity. While this is not desirable at high velocities, it is required at low velocities in order to allow precise hovering. If the measurements of the lateral velocity are accurate enough for both high and low flight

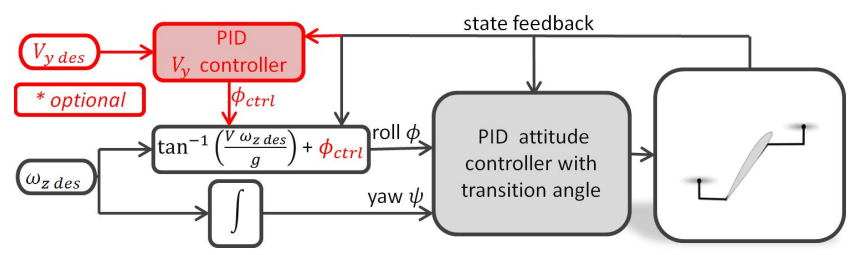

Fig. 15: Overview of the $\omega_{z}$ controller with optional control of $V_{y}$.

speeds, a $V_{y}$ controller can regulate the lateral velocity that is requested by the user by adapting the additional roll angle. For the test vehicle described in this paper, the user directly applies an additional roll angle to the angle commanded by the controller to control the lateral velocity.

\section{FLIGHT TEST AND VALIDATION}

The proposed control approach in this paper is implemented on the test vehicle. First the intuitive attitude control approach is implemented. To this end an extra channel on the remote control is used by the operator to turn the reference frame of the vehicle and allow intuitive operation. This controller is then used to collect data for the velocity controller: throttle and transition angle are set and kept constant until the vehicle reaches a steady state. $V_{x}$, measured by GPS, and $V_{z}$, measured by barometer, are logged on the flight controller of the vehicle and used to validate the simulations as was illustrated in figure 13 in section 3.

As a first step to validate the velocity control approach, the polynomial fits are used in feed forward without a feedback control loop. The throttle and transition angle commands that are generated by the feed forward controller during a test flight are presented in figure 16 for the case in which the test vehicle starts from hover at a constant altitude and the user commands cruise speed $V_{x}$, presented by the blue line. The vehicle transitions forward and throttle is kept constant since this is required at cruise speed.

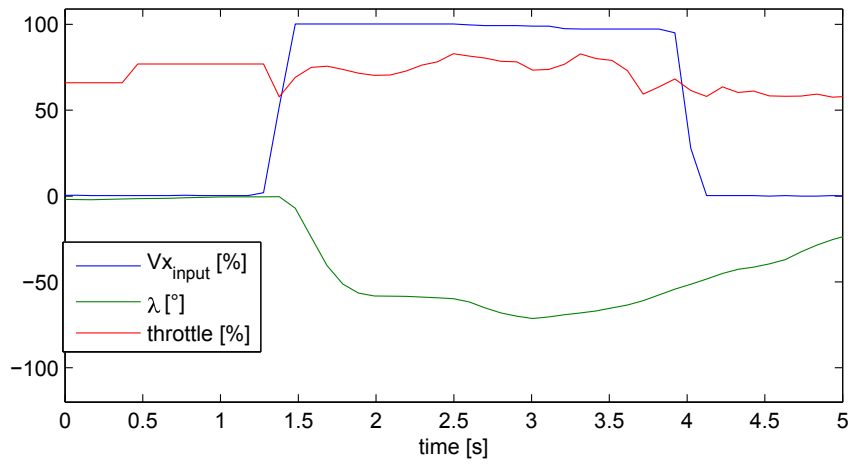

Fig. 16: Visualisation of the feed forward horizontal velocity controller on the test vehicle, starting from hover: an operator request to fly forward is realised by decreasing pitch. 
Figure 17 shows the logged data during a test flight in which the command for $V_{x}$ is kept constant and the $V_{z}$ command is varied. Initially the test vehicle is flying in steady state at a transition angle of $30^{\circ}$ or a pitch angle of the body frame with respect to the earth of $-30^{\circ}$, presented by the green line. When the user commands a high $V_{z}$, presented by the blue line, both throttle and pitch are increased. For a desired descend rate, the vehicle pitches further nose down and throttle is decreased.

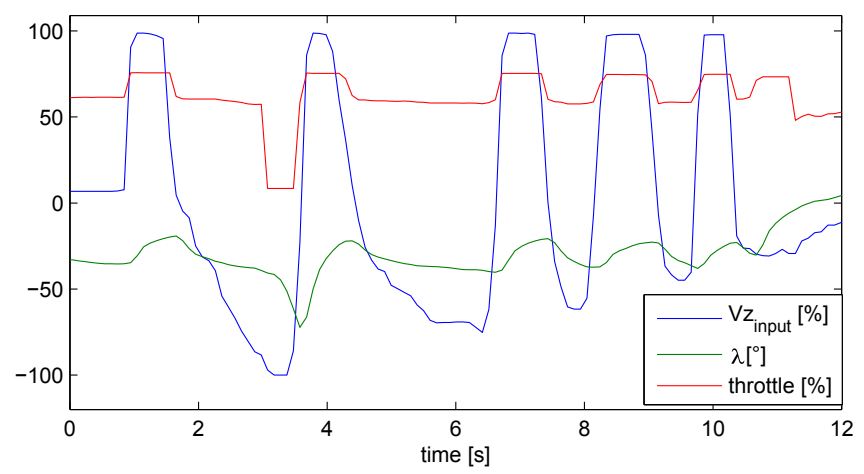

Fig. 17: Visualisation of the feed forward vertical velocity controller on the test vehicle, flying at $30^{\circ}$ transition angle: an operator request to climb is realised by increasing transition angle and throttle, a request to descend does the opposite.

During testing, a problem occurred for descending at high speed. The vehicle starts to accelerate towards the ground but the propulsion system is not able any more to generate enough moment to pitch the vehicle back up and stabilize the descent. Although the motor can spin the propeller to more than $9000 R P M$ (see figure 9, section 3) and the propeller generates thrust to speeds up to $25 \mathrm{~m} / \mathrm{s}$ at this $R P M$ (figure 10 , section 3 ), the moment to pitch the vehicle back up in this fast descent is too low. There are several ways to overcome this dangerous problem. A propeller with a higher pitch can be chosen, or the maximum $R P M$ of the motors can be increased by selecting motors with a higher $k V$ value or using a battery with a higher voltage. Both options result in more available thrust at higher speeds, but also a loss in efficiency at low speeds. Increasing the length of the arms to which the motors are mounted increases the control moment of the propulsion system with differential thrust without any loss of efficiency, however this leads to a heavier structure. A last option that does not result in an efficiency loss or heavy structure, is to use motor controllers that allow the propeller to spin backwards and produce a negative thrust. This way, control moment is available at any speed and additionally the vehicle will be able to 'brake'.

\section{CONCLUSION AND FUTURE WORK}

The control approach described in this paper offers a general solution for the control of various configurations of transitioning VTOL UAV controlled by differential thrust. The control approach works between hover and cruise or vice versa through the continuous transition. In a first step, the addition of a rotating reference frame allows a human operator to control the vehicle with an intuitive attitude controller. Secondly, a velocity controller controls the throttle and transition angle of the vehicle to accomplish a desired horizontal and vertical flight speed. As a result, an inexperienced human operators or a general path planning algorithm is able to control any VTOL UAV controlled by differential thrust in an intuitive manner. To apply this controller to a given VTOL UAV, flight characteristics of the vehicle are simulated, based on the specific configuration of the VTOL UAV and are validated through a small series of test flights. The velocity controller relies on a stable attitude controller for all flight phases. It could therefore be necessary to apply gain scheduling.

A test vehicle was custom built in order to test and validate the control approach. Data from flight tests with the intuitive attitude control were used to validate a simulation model, since obtaining enough test data points in the whole operating range of the VTOL UAV has proven to be very time consuming. The simulation model of the VTOL UAV provided a solution to obtain these data points. The data points that were measured during test flight match the simulated points with errors less than $20 \%$. A way to improve the data used to generate the polynomials, is using flight test data instead of simulated data. Since the number of possible operating points is large, this test process could be automated. It would also be possible to update the polynomials during flight, if a consistent error is measured for a certain required operating point.

From flight tests we learned that the current test vehicle cannot recover from a fast descending flight regime. The best proposed solution for this problem is to implement a propulsion system that allows the propeller to turn in the opposite direction in order to brake and keep control of the vehicle in all situations.

In future work, a wind speed estimator has to be added to distinguish between air speed and ground speed so that a velocity with respect to the ground can be controlled. The control approach has shown to be promising, although further testing on different platforms is required to show its general applicability.

\section{ACKNOWLEDGEMENTS}

A special thanks to Cyriel Notteboom, who constructed the test vehicle and model airplane club "The Flying Moths", who allowed us to use their field for tests. 


\section{REFERENCES}

[1] R. Hugh Stone, Peter Anderson, Colin Hutchison, Allen Tsai, Peter Gibbens, and KC Wong. Flight testing of the t-wing tail-sitter unmanned air vehicle. Journal of Aircraft, 45(2):673-685, 2008.

[2] Vikram Hrishikeshavan, Dean Bawek, Omri Rand, and Inderjit Chopra. Development of transition control methodology for a quad rotor-biplane micro air vehicle from hover to forward flight. Abstract submitted for the AHS International Specialists Meeting on Unmanned Rotorcraft Scottsdale, AZ, January 2013.

[3] Charles Manning JD Claridge. 2015 xcraft enterprises http://xcraft.io/ last visit: 2016-01-08.

[4] Atsushi Oosedo, Atsushi Konno, Takaaki Matsumoto, Kenta Go, Koji Masuko, and Masaru Uchiyama. Design and attitude control of a quad-rotor tail-sitter vertical takeoff and landing unmanned aerial vehicle. Advanced Robotics, 26(3-4):307-326, 2012.

[5] Brasil Santos Labs, Rio de Janeiro. Annular wing quadrocopter demonstrated at laad defence and security expo, 2013.

[6] Matthew Anderson, Kai Lehmkuehler, Derrick Ho, KC Wong, and Patrick Hendrick. Propeller location optimisation for annular wing design.

[7] Adnan S Saeed, Ahmad Bani Younes, Shafiqul Islam, Jorge Dias, Lakmal Seneviratne, and Guowei Cai. A review on the platform design, dynamic modeling and control of hybrid uavs. In Unmanned Aircraft Systems (ICUAS), 2015 International Conference on, pages 806-815. IEEE, 2015.

[8] Guadalupe Flores and Rogelio Lozano. Transition flight control of the quad-tilting rotor convertible mav. In Unmanned Aircraft Systems (ICUAS), 2013 International Conference on, pages 789-794. IEEE, 2013.

[9] M. Hochstenbach and C. Notteboom. Ontwerp en bouw van een onbemand vliegtuig voor autonoom pakkettransport met gecontroleerde transitie van verticaal opstijgen naar voorwaartse vlucht. Master's thesis, KU Leuven, 2014.

[10] Bart Theys, Cyriel Notteboom, Menno Hochstenbach, and Joris De Schutter. Design and control of an unmanned aerial vehicle for autonomous parcel delivery with transition from vertical take-off to forward flight. International Journal of Micro Air Vehicles, 7(4):395405, 2015.

[11] Eric N Johnson, Michael A Turbe, Allen D Wu, Suresh K Kannan, and James C Neidhoefer. Flight test results of autonomous fixed-wing uav transitions to and from stationary hover. In Proceedings of the AIAA Guidance, Navigation, and Control Conference Exhibit, Monterey, CO, 2006.

[12] C. De Wagter, D. Dokter, G. de Croon, and B. Remes. Multi-lifting-device uav autonomous flight at any transition percentage. In EuroGNC 2013, 2013.

[13] Peter Ferrell, Brian Smith, Brandon Stark, and YangQuan Chen. Dynamic flight modeling of a multimode flying wing quadrotor aircraft. In Unmanned Aircraft Systems (ICUAS), 2013 International Conference on, pages 398-404. IEEE, 2013.

[14] Takahito Mikami and Kenji Uchiyama. Design of flight control system for quad tilt-wing uav. In Unmanned Aircraft Systems (ICUAS), 2015 International Conference on, pages 801-805. IEEE, 2015.

[15] Satoshi Kohno and Kenji Uchiyama. Design of robust controller of fixed-wing uav for transition flight. In $\mathrm{Un}$ manned Aircraft Systems (ICUAS), 2014 International Conference on, pages 1111-1116. IEEE, 2014.

[16] E.J.J. Smeur. Modelling of a hybrid uav using test flight data. Master's thesis, TU Delft, April 2014.

[17] Chris C. Critzos, Harry H. Heyson, and Robert W. Boswinkle. Aerodynamic characteristics of naca 0012 airfoil section at angles of attack from 0 to 180 . Technical report, National advisory committee for aeronautics, Washington, January 1955.

[18] APC Advanced Presicion Composites. Apc propeller performance data.

[19] Rogelio G Hernandez-Garcia and H Rodriguez-Cortes. Transition flight control of a cyclic tiltrotor uav based on the gain-scheduling strategy. In Unmanned Aircraft Systems (ICUAS), 2015 International Conference on, pages 951-956. IEEE, 2015.

[20] Beard and McLain. Small Unmanned Aircraft. Princeton University Press, 2012. 